\title{
Self Assembly Peptides- Biomimetic Material
}

\section{Malini V*}

Department of Preventive and Pediatric Dentistry, Kerala University of Health Sciences, India

*Corresponding author: Malini Venugopal, Consultant Pedodontist, Department of Preventive and Pediatric Dentistry, Kerala University of Health Sciences, Kerala, India, Email: mailtomalu85@gmail.com

\section{Review Article}

Volume 4 Issue 2

Received Date: April 30, 2019

Published Date: May 14, 2019

DOI: $10.23880 /$ oajds-16000227

\section{Abstract}

Self-assembling peptides are peptides which undergo spontaneous assembling into ordered nanostructures. As dentistry we are moving towards minimal invasive methods and hence there is a growing interest with regard to this new material. Self assembling peptides can prevent dental caries at an early stage thus preventing surgical process of cavity preparation and restorations.

Keywords: Peptides; Biomimetics; Remineralization

\section{Introduction}

Dental Caries is the most common disease worldwide. Inspite of advancement in technologies caries remains the single most widespread disease of the childhood. It is now well known that Dental caries is not a result of a single acid formed attack but it is caused due to imbalance between demineralization and remineralization cycle occurring in oral cavity. For early carious lesions, current evidence suggests that the use of noninvasive and nonrestorative methods like sealants and resin infiltration, remineralization procedures mainly with fluorides and CPP - ACP (casein phosphopeptide amorphous calcium phosphate) vehicles are better than invasive procedures. Biomimetic self-assembling peptide, P11-4, nucleated hydroxyapatite are new development and are apparently capable of in situ enamel regeneration following infiltration into caries-like lesions [1].

\section{Caries Balance}

Enamel caries starts by subsurface demineralization, leaving a porous mineral surface covering the lesion body. During the demineralization process, calcium phosphate minerals, making up most of the enamel structure, is dissolved due to acidic $\mathrm{pH}$ and results in pores between crystallites. During remineralization, calcium phosphate supersaturated saliva redeposit minerals either on existing crystallites or triggers de novo formation of crystallites. This presents the natural regeneration process of the enamel tissue [2]. It is only after demineralization of approximately $30 \%$, the mineralized surface collapses and breaks irreversibly. Biofilm removal and diet control to some extent support natural remineralization of the carious lesions via $\mathrm{Ca} 2+$ from saliva but it is restricted to the outer $\sim 30 \mu \mathrm{m}$ of the tooth enamel that is marginally affected by caries. Infiltration of lesion pores using low viscosity resins such as sealants can halt caries progression as it provides a barrier to diffusion within the lesion $[3,4]$.

The use of biomimmetic material self-assembling peptide P11-4 have shown to regenerate demineralized tooth tissue on smooth surfaces. Traditional materials lacked the ability to integrate with biological systems through a cellular pathway which resulted in the failure of these material. A true regenerative approach, aiming at 


\section{Open Access Journal of Dental Sciences}

regenerating hydroxyapatite crystals within the subsurface is needed [5].

P11 4 is a bioactive peptide. It assemble into a three dimensional fibrillar scaffold in response to environmental conditions of $\mathrm{pH}$. Hence $\mathrm{P} 11-4$ is considered as a rationally-designed self-assembling peptide. It is synthesised from natural amino acids. Selfassembling peptides undergo well-characterized hierarchical self-assembly. It forms a new generation of well-defined biopolymers which will prevent the progression of dental caries. It is known to form tapes and ribbons within seconds, and fibrils and fibers within the following $24 \mathrm{hrs}$.

Caries lesions shown negative charges and P11-4 switches from a low viscosity isotropic liquid to an elastomeric pneumatic gel at $\mathrm{pH}<7.4$ and in the presence of cations. P11-4 self-assemble with the anionic groups of other P11-4 side chains and attract $\mathrm{Ca}++$ ions, inducing de novo precipitation of hydroxyapatite. A number of in vivo and in vitro experiments, have shown that the assembled P11-4 fibers to be highly biocompatible with low immunogenicity [6].

The earliest clinical sign of enamel caries is the appearance of a 'white spot' lesion on the tooth surface. Clinicians usually monitor the lesion appearance and use topical fluorides and determine whether the lesion will progress. If it progresses restorations would are given. Non-surgical intervention promoting biomineralization or regeneration at the white spot lesion stage can eliminate the need for a surgical excavation of the tooth and placement of a restoration [7]. Infiltration of early caries lesions using low viscosity monomeric P11-4 would result in triggered self-assembly within the lesion would create a subsurface bioactive scaffold capable of recapitulating normal histogenesis by mineral deposition in situ.

Peptide treatment shows significant increase in the net mineral gain due to a combined effect of deposition of mineral and inhibition of mineral loss. P11-4 have a high affinity to hydroxyapatite and it has a potential to nucleate hydroxyapatite. The surface $\mathrm{Ca}^{2+}$ binding sites correspond exactly with the spacing of the $\mathrm{Ca}^{2+}$ ions in the hydroxyapatite crystal lattice. Self assembling peptide is known to form a 3D matrix within demineralized carious lesions forming denovo hydroxyapatite crystal. These peptides have shown encouraging results as a scaffold for enamel regeneration $[8,9]$.

In a randomized, controlled, single-blinded study, the safety and clinical efficacy of P11-4 in treatment of initial caries were evaluated using a randomized controlled trial (RCT) design. No adverse events, medical complications, or allergic reactions related to the treatments were reported. Clinical applicability was reported as easier than placing a filling or a sealant. All patients were willing to have future noninvasive treatments. In vivo and in vitro experiments, the assembled P11-4 fibers were shown to be highly biocompatible with low immunogenicity. In a study remineralization of natural carious lesions in human teeth, were monitored using the Canary System. Canary Number of 40-50 (early carious lesions) remained basically unchanged for both control groups, but it reduced to values only slightly higher than that of sound enamel for the P11-4 group showing the remineralizing capacity of self assembling peptide P11-4 [10].

\section{Conclusion}

Self-assembling peptide P11-4 is a simple, safe, and effective noninvasive treatment for early carious lesions, and can be regarded as superior to the present clinical gold standard of fluoride treatment alone. However more clinical studies may be needed to completely understand the material.

\section{References}

1. Quock RL (2015) Dental Caries: A Current Understanding and Implications. Journal of Nature and Science 1(1): 27.

2. Featherstone JD (2004) The caries balance: the basis for caries management by risk assessment. Oral health \& preventive dentistry 2(1): 259-264.

3. Johnson AR (1975) The early carious lesion of enamel. J Oral Pathol 4(3): 128-157.

4. Fan PL, Seluk L W, O Brien WJ (1975) Penetrativity of sealants: I. J Dent Res 54(2): 262-264.

5. Kirkham J, Firth A, Vernals D, Boden N, Robinson C, et al. (2007) Self-assembling peptide scaffolds promote enamel remineralization. Journal of dental research 86(5): 426-430.

6. Brunton PA, Davies R, Burke (2013) Treatment of early caries lesions using biomimetic self-assembling peptides-a clinical safety trial. Br Dent J 215(4): 1-6.

7. Robinson C, Brookes SJ, Kirkham J, Wood SR, Shore RC (2001) In vitro studies of the penetration of adhesive resins into artificial caries-like lesions. Caries Res 35(2): 136-141. 


\section{Open Access Journal of Dental Sciences}

8. Paris S, Meyer Lueckel H (2010) Inhibition of caries progression by resin infiltration in situ. Caries Res 44(1): 47-54.

9. Paris S, Hopfenmuller W, Meyer Lueckel H (2010) Resin infiltration of caries lesions: an efficacy randomized trial. J Dent Res 89: 823-882.
10. Alkilzy M, Tarabaih A, Santamaria RM, Splieth $\mathrm{CH}$ (2018) Self-assembling peptide P11-4 and fluoride for regenerating enamel. J Dent Res 97(2): 148-154. 